\title{
Determination of the nucleus size from the growth probability of clusters
}

\author{
Joop H. ter Horst ${ }^{\text {a) }}$ \\ Laboratory for Process Equipment, Delft University of Technology, Leeghwaterstraat 44, \\ 2628 CA Delft, The Netherlands \\ Dimo Kashchiev \\ Institute of Physical Chemistry, Bulgarian Academy of Sciences, ul. Acad. G. Bonchev 11, \\ Sofia 1113, Bulgaria
}

(Received 4 March 2003; accepted 1 May 2003)

\begin{abstract}
Exact and approximate general expressions for the growth and decay probabilities of a cluster in one-component nucleation are given. A method is proposed for a model-independent determination of the nucleus size with the help of data for the dependence of the cluster growth probability on the cluster size. Monte Carlo simulation data for the cluster growth probability are obtained for homogeneous one-component nucleation of droplets in Lennard-Jones vapors. From these data, the size of the nucleus droplet and the Zeldovich factor are determined by the newly proposed method. (c) 2003 American Institute of Physics. [DOI: 10.1063/1.1585020]
\end{abstract}

\section{INTRODUCTION}

Nucleation is known to occur by formation of molecular (or atomic) clusters of various size $n=1,2,3, \ldots$. A given cluster of $n$ molecules (or atoms) changes randomly its size as a result of successive attachments and detachments of single molecules (or atoms) to and from it. The cluster of size $n=n^{*}$ is the nucleus (called also critical nucleus). It is that particular cluster to and from which molecules are attached and detached with equal frequency. ${ }^{1}$ The subnuclei are the clusters of size $n<n^{*}$ - they tend to decay, because per unit time less molecules are attached to than detached from them. On the contrary, the supernuclei (the clusters of $n>n^{*}$ molecules) are characterized by attachment frequencies greater than the detachment ones and for that reason most of them are capable of growth up to macroscopic sizes.

As molecular attachments and detachments are random events, a given $n$-sized cluster can grow and reach a previously specified sufficiently large supernucleus size only with a certain probability $P(n)$. Using a result of probability theory, ${ }^{2}$ White ${ }^{3}$ showed that $P(n)$ can be represented exactly in terms of the ratio between the stationary and equilibrium cluster size distributions. These distributions, however, depend on the nucleus size $n^{*}$ which is an important parameter in nucleation theory. Thus, knowing $P$ as a function of $n$ allows obtaining information about $n^{*}$ under the particular nucleation conditions.

The growth probability $P$ is hard to measure experimentally, because the subnuclei, the nucleus and the smallest supernuclei are nanoscopically small particles. At the same time, $P$ is readily obtainable by computer simulations which have already become a powerful technique to study the nucleation behavior of supersaturated systems. ${ }^{4-17}$ To the best of our knowledge, however, in computer simulations so far $P(n)$ has not been studied and analyzed with the aim to

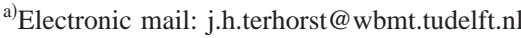

obtain data for the nucleus size $n^{*}$ and its dependence on the supersaturation.

The objective of the present paper is to demonstrate how $P(n)$ can be used for a model-independent determination of $n^{*}$. The method outlined is particularly suitable for computer simulations of nucleation, because it allows obtaining the supersaturation dependence of $n^{*}$ with the use of a relatively short computer time. Our analysis is restricted to onecomponent nucleation at constant supersaturation.

\section{CLUSTER GROWTH AND DECAY PROBABILITIES}

We consider a supersaturated one-component gaseous, liquid or solid phase which is kept at constant absolute temperature $T$ and supersaturation $\Delta \mu$. For such a system, when the clusters of the new phase attach and detach only single molecules, the stationary and equilibrium cluster size distributions are related by the exact formula ${ }^{18}(2 \leqslant n \leqslant M-1)$

$$
\begin{aligned}
X(n)= & C(n)\left[1+\sum_{i=2}^{M-1} g_{2} g_{3} \cdots g_{i} / f_{2} f_{3} \cdots f_{i}\right]^{-1} \\
& \times \sum_{i=n}^{M-1} g_{2} g_{3} \cdots g_{i} / f_{2} f_{3} \cdots f_{i},
\end{aligned}
$$

which is valid for any kind of nucleation-homogeneous, heterogeneous, three-dimensional or two-dimensional. Here $X(n)$ and $C(n)$ are, respectively, the stationary and equilibrium concentrations of $n$-sized clusters, $f_{n}$ and $g_{n}$ are the frequencies of attachment and detachment of molecules to and from an $n$-sized cluster, respectively, and $M>n^{*}$ is the size of such large supernucleus that is capable of practically irreversible overgrowth.

A given $n$-sized cluster has a probability $P(n)$ to grow up to the supernucleus size $n=M$ and a probability $Q(n)$ to decay to the monomer size $n=1$. These two probabilities are related by

$$
P(n)+Q(n)=1,
$$




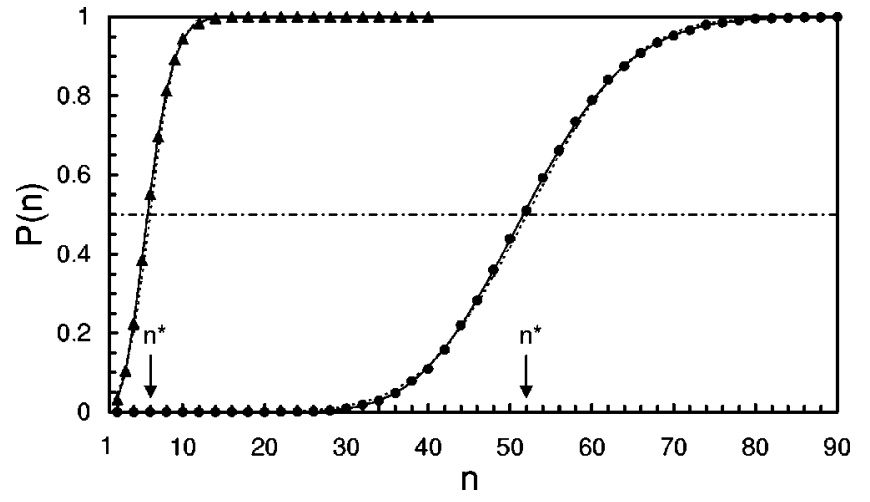

FIG. 1. Dependence of the cluster growth probability on the cluster size: Circles and triangles-numerical simulation data for homogeneous nucleation of water droplets in steam at $T=293 \mathrm{~K}$ and $S=4.5$ and 22, respectively; solid curves-exact Eq. (10); dotted curves—approximate Eq. (18).

and are entirely determined by the attachment and detachment frequencies $f_{n}$ and $g_{n}$. As an example, shown in Fig. 1 is the dependence of the cluster growth probability $P$ on the cluster size $n$ in the case of homogeneous nucleation of water droplets in steam at $T=293 \mathrm{~K}$. The circles and the triangles depict $P(n)$ for supersaturation ratios $S \equiv p / p_{\mathrm{e}}=4.5$ and 22, respectively. The $P(n)$ dependence depicted by these symbols is obtained by numerical simulation of the changes occurring randomly in the size of the water clusters as a result of attachment and detachment of single molecules with frequencies ${ }^{18}(1 \leqslant n \leqslant M-1)$

$$
\begin{aligned}
f_{n}= & {\left[p /\left(2 \pi m_{0} k T\right)^{1 / 2}\right]\left(36 \pi / \rho^{2}\right)^{1 / 3} n^{2 / 3}, } \\
g_{n}= & {\left[p_{\mathrm{e}} /\left(2 \pi m_{0} k T\right)^{1 / 2}\right]\left(36 \pi / \rho^{2}\right)^{1 / 3}(n} \\
& -1)^{2 / 3} \exp \left[2\left(36 \pi / \rho^{2}\right)^{1 / 3} \gamma / 3 k T n^{1 / 3}\right] .
\end{aligned}
$$

Here $p$ and $p_{\mathrm{e}}=2.3 \mathrm{kPa}$ are the actual and equilibrium pressures of the steam, $m_{0}=3 \times 10^{-26} \mathrm{~kg}$ is the mass of a water molecule, $\rho=33.3 \mathrm{~nm}^{-3}$ is the molecular density of water at water-steam coexistence, $\gamma=73 \mathrm{~mJ} / \mathrm{m}^{2}$ is the specific surface energy of the planar water-steam interface, and $k$ is the Boltzmann constant. The nucleus size $n^{*}=52$ and 6 corresponding to the above $S$ values, respectively, is calculated from the Gibbs-Thomson equation ${ }^{18}$

$$
n^{*}=32 \pi \gamma^{3} / 3 \rho^{2}(k T \ln S)^{3} \text {. }
$$

In the numerical simulation the $n$-sized water cluster is allowed to gain a molecule and become an $(n+1)$-sized cluster with probability $(1 \leqslant n \leqslant M-1)$

$$
p_{n}=f_{n} /\left(f_{n}+g_{n}\right),
$$

or to lose a molecule and become an $(n-1)$-sized cluster with probability $(1 \leqslant n \leqslant M-1)$

$$
q_{n}=g_{n} /\left(f_{n}+g_{n}\right) .
$$

If for a given cluster size the attachment probability calculated from Eq. (6) is greater than or equal to a randomly generated number between 0 and 1 , the cluster size is increased by one molecule. Respectively, if this probability is smaller than the random number, the cluster size is decreased by one molecule. The largest size $M$ to which the $n$-sized cluster grows is set equal to 500, because the resulting $P(n)$

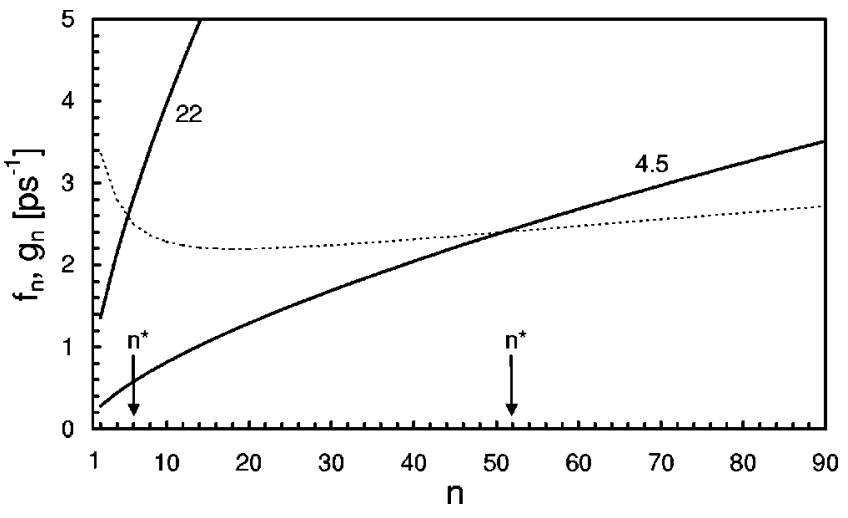

FIG. 2. Dependence of the cluster attachment $\left(f_{n}\right)$ and detachment $\left(g_{n}\right)$ frequencies on the cluster size for homogeneous nucleation of water droplets in steam at $T=293 \mathrm{~K}$ : Solid curves $-f_{n}$ from Eq. (3) at $S=4.5$ and 22 (as indicated); dotted curve $-g_{n}$ from Eq. (4) ( $g_{n}$ is $S$-independent).

function does not depend on the chosen $M$ value as long as $M \gg n^{*}$. For each cluster size $n$, the symbols in Fig. 1 represent the average $P(n)$ value obtained from a single run consisting of 10000 simulations. For all $P(n)$ values, the standard deviation calculated from 20 such runs is smaller than the symbols in Fig. 1.

The solid curves in Fig. 2 exhibit the dependence (3) of $f_{n}$ on $n$ at $S=4.5$ and 22 (as indicated), and the dotted curve depicts the $g_{n}$ dependence (4). As seen, molecules are attached to and detached from the nucleus droplet with practically equal frequencies $f_{n *}=2.44 \mathrm{ps}^{-1}$ and $g_{n *}=2.41 \mathrm{ps}^{-1}$ at $n^{*}=52$ and $f_{n^{*}}=2.83 \mathrm{ps}^{-1}$ and $g_{n *}=2.50 \mathrm{ps}^{-1}$ at $n^{*}$ $=6$. Whereas molecular attachment to the subnuclei (the $n$ $<n^{*}$-sized droplets) is less frequent than detachment from them, the opposite is true for the supernuclei (the $n>n^{*}$-sized droplets). For that reason the growth probability $P$ of the subnuclei and the supernuclei is, respectively, smaller or greater than 1/2 (see Fig. 1). According to Fig. 1, and as found also by White, ${ }^{3}$ the nucleus has an almost equal chance to grow to macroscopic size and to decay to a monomer: $P\left(n^{*}\right)=0.51$ at $n^{*}=52$ and $P\left(n^{*}\right)=0.55$ at $n^{*}=6$. The reason for the slight departure of $P\left(n^{*}\right)$ from $1 / 2$ is (i) the asymmetry of the frequencies $f_{n}$ and $g_{n}$ around $n=n^{*}$ and (ii) the inequality in the length of the paths traveled by the nucleus on the size axis when it decays to $n=1$ and when it grows to $n=M$ (in the numerical simulation $n^{*}-1=51$, $M-n^{*}=448$ when $n^{*}=52$, and $n^{*}-1=5, M-n^{*}=494$ when $n^{*}=6$ ). When $f_{n}$ and $g_{n}$ are strictly symmetrical with respect to $n=n^{*}$ and when $n^{*}-1=M-n^{*}$, the nucleus has equal chance for growth or decay, i.e., then $P\left(n^{*}\right)=1 / 2$.

According to probability theory, ${ }^{2}$ the cluster decay probability $Q(n)$ is given by the exact formula $(2 \leqslant n \leqslant M-1)$

$$
\begin{aligned}
Q(n)= & {\left[1+\sum_{i=2}^{M-1} g_{2} g_{3} \cdots g_{i} / f_{2} f_{3} \cdots f_{i}\right]^{-1} } \\
& \times \sum_{i=n}^{M-1} g_{2} g_{3} \cdots g_{i} / f_{2} f_{3} \cdots f_{i},
\end{aligned}
$$

which, in view of Eq. (1), can be rewritten simply as ${ }^{3}$

$$
Q(n)=X(n) / C(n) .
$$


Combining Eqs. (1), (2), (8), and (9), we find that the growth probability $P(n)$ of an $n$-sized cluster is given exactly by $(2 \leqslant n \leqslant M-1)$

$$
\begin{aligned}
P(n)= & 1-\left[1+\sum_{i=2}^{M-1} g_{2} g_{3} \cdots g_{i} / f_{2} f_{3} \cdots f_{i}\right]^{-1} \\
& \times \sum_{i=n}^{M-1} g_{2} g_{3} \cdots g_{i} / f_{2} f_{3} \cdots f_{i},
\end{aligned}
$$

or, in terms of $X(n)$ and $C(n)$, by

$$
P(n)=1-X(n) / C(n) .
$$

It is important to bear in mind that the above four formulas are valid for any kind of nucleation-homogeneous, heterogeneous, three-dimensional, or two-dimensional.

The solid curves in Fig. 1 display the exact $P(n)$ dependence (10) calculated numerically with the help of $f_{n}$ and $g_{n}$ from Eqs. (3) and (4) for water droplets nucleated homogeneously in steam under the conditions noted above. As seen, Eq. (10) is in perfect agreement with the $P(n)$ dependence resulting from the numerical simulation.

Clearly, Eqs. (9) and (11) can be used for analysis of $Q(n)$ and $P(n)$ data only after expressing the $X(n) / C(n)$ ratio as an explicit function of $n$. It turns out that this can be done at the expense of certain approximations, but without any loss of generality. Namely, we can consider $n$ as a continuous variable, set $M=\infty$ and employ the following approximation $^{18}(1 \leqslant n<\infty)$

$X(n)=C(n)\left\{1-\operatorname{erf}\left[\beta\left(n-n^{*}\right)\right]\right\} /\left\{1-\operatorname{erf}\left[\beta\left(1-n^{*}\right)\right]\right\}$.

Here the numerical factor $\beta>0$ is defined by

$$
\beta=\left[-\left(\mathrm{d}^{2} W / \mathrm{d} n^{2}\right)_{n=n *} / 2 k T\right]^{1 / 2},
$$

where $W(n)$ is the work to form an $n$-sized cluster. As known, ${ }^{18} \beta$ is related to the width $\Delta^{*}$ of the nucleus region and to the Zeldovich factor $z$ by

$$
\beta=\pi^{1 / 2} / \Delta^{*}=\pi^{1 / 2} z .
$$

Substitution of $X(n)$ from Eq. (12) into Eqs. (9) and (11) yields $(1 \leqslant n<\infty)$

$$
Q(n)=\left\{1-\operatorname{erf}\left[\beta\left(n-n^{*}\right)\right]\right\} /\left\{1-\operatorname{erf}\left[\beta\left(1-n^{*}\right)\right]\right\},
$$

for the decay probability of an $n$-sized cluster and $(1 \leqslant n$ $>\infty)$

$$
\begin{aligned}
P(n)= & \left\{\operatorname{erf}\left[\beta\left(n-n^{*}\right)\right]-\operatorname{erf}\left[\beta\left(1-n^{*}\right)\right]\right\} /\{1-\operatorname{erf}[\beta(1 \\
& \left.\left.\left.-n^{*}\right)\right]\right\},
\end{aligned}
$$

for the growth probability of such a cluster. To a very good accuracy these two equations can be used in the simpler form $(1 \leqslant n<\infty)$

$$
\begin{aligned}
& Q(n)=(1 / 2)\left\{1-\operatorname{erf}\left[\beta\left(n-n^{*}\right)\right]\right\}, \\
& P(n)=(1 / 2)\left\{1+\operatorname{erf}\left[\beta\left(n-n^{*}\right)\right]\right\},
\end{aligned}
$$

when $\beta$ and $n^{*}$ obey the condition

$$
\beta\left(n^{*}-1\right)>1,
$$

since then $\operatorname{erf}\left[\beta\left(1-n^{*}\right)\right] \approx-1$ with an error of less than $15 \%$. In practice, the above inequality is almost always satisfied, because it is equivalent to the condition $W^{*} / k T>3$ (Ref. $18), W^{*} \equiv W\left(n^{*}\right)$ being the work done to form the nucleus. We emphasize that, like the exact Eqs. (8)-(11), though approximate, Eqs. (15)-(18) are general formulas valid for any kind of nucleation.

The dotted curves in Fig. 1 display the approximate $P(n)$ dependence (18) with $n^{*}=52, \beta=0.0694$ and $n^{*}=6$, $\beta=0.293$. These values correspond to the case considered above of homogeneous nucleation of water droplets in steam at $T=293 \mathrm{~K}$ and $S=4.5$ and 22 , respectively. The $\beta$ values are calculated from the expression

$$
\beta=\left(\ln S / 6 n^{*}\right)^{1 / 2}
$$

given by the classical theory of homogeneous nucleation. ${ }^{18}$ As evidenced in Fig. 1, in the practically interesting range of $P>0.01$ the $P(n)$ dependence (18) is a very good approximation to the exact $P(n)$ dependence (10).

As predicted by the approximate Eq. (18), and seen in Fig. 1 as well, $P\left(n^{*}\right)=1 / 2$ in a slight disagreement with the $P\left(n^{*}\right)$ values of 0.51 and 0.55 from the numerical simulation data (the symbols in Fig. 1) or the exact $P(n)$ dependence (10) (the solid curves in Fig. 1). This result is of great practical importance, for it allows a sufficiently good approximate determination of the nucleus size $n^{*}$ from $P(n)$ data obtained, e.g., by Monte Carlo or molecular dynamics simulations. Indeed, when such data are available, $n^{*}$ is readily determined from the condition

$$
P\left(n^{*}\right)=1 / 2 .
$$

Naturally, a determination of $n^{*}$ and, in addition, of $\beta$ can be done when these are considered as free parameters in Eq. (18). A best fit of $P(n)$ from Eq. (18) to the simulation $P(n)$ data then yields both $n^{*}$ and $\beta$.

To exemplify this procedure we can apply Eqs. (18) and (21) to the numerical simulation data in Fig. 1. As seen from the intersection of the dash-dotted line with the solid curves in the figure, Eq. (21) leads to $n^{*}=51.6$ and 5.7 rather than to the exact $n^{*}=52$ and 6, respectively. The best fit of Eq. (18) to the same data gives $n^{*}=52.8, \beta=0.0698$ and $n^{*}$ $=5.8, \beta=0.294$ instead of the exact $n^{*}=52, \beta=0.0694$, and $n^{*}=6, \beta=0.293$, respectively. We see that the fitting results in $n^{*}$ values only slightly different from those determined by means of Eq. (21). Hence, Eq. (21) appears as a valuable tool for the express and quite accurate determination of $n^{*}$ from $P(n)$ data (the error may be within a molecule or two only). Moreover, this determination is modelindependent, because Eq. (21) applies to any kind of nucleation. When $n^{*}$ is determined in such a way for different values of the supersaturation $\Delta \mu$, it is possible to use the resulting $n^{*}(\Delta \mu)$ dependence for confrontation with a known theoretical dependence such as the classical GibbsThomson equation. A good agreement with this equation would allow evaluation of the specific surface or edge energy of the nucleus in one-component homogeneous or heterogeneous three- or two-dimensional nucleation, respectively. 


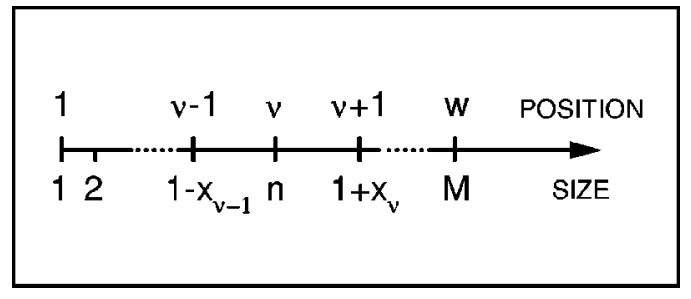

FIG. 3. Size interval $[1, M]$ divided into $w-1$ subintervals.

\section{COMPUTATIONAL APPROACH}

Given a sufficiently long simulation time, a cluster of initial size $n$ either decays to size 1 or grows to a large enough supernucleus size $M$ and this allows the determination of the decay and growth probabilities $Q(n)$ and $P(n)$, respectively. However, computer time is limited and in order to be saved, it is convenient to divide the interval $[1, M]$ in which the cluster performs its random walk into $w-1$ subintervals $(w=2,3, \ldots, M)$ with known equal or different lengths $x_{\nu}\left(1 \leqslant x_{\nu} \leqslant M-1 ; \nu=1,2, \ldots, w-1\right)$. In this way, as seen in Fig. 3, the cluster size axis is transformed into a position axis on which positions $1,2, \ldots, \nu-1, \nu, \nu+1, \ldots w$ $-1, w$ correspond to sizes $1,1+x_{1}, \ldots n-x_{\nu-1}, n, n$ $+x_{\nu}, \ldots M-x_{w-1}, M$ of the cluster, respectively. We can thus regard the $n$-sized cluster in position $\nu$ as performing a random walk on the position axis with probabilities $\lambda_{\nu}$ and $\mu_{\nu}$ for forward transition from position $\nu$ to position $\nu+1$ and backward transition from position $\nu$ to position $\nu-1$, respectively. The transition probabilities $\lambda_{\nu}$ and $\mu_{\nu}$ can be obtained by simulation provided the number of the simulation runs with the corresponding $n$-sized cluster is sufficiently large and the runs themselves are long enough. Indeed, let $N_{\nu}^{+}$be the number of runs in which the $n$-sized cluster grows to size $n+x_{\nu}$ before decaying to size $n$ $-x_{\nu-1}$, i.e., reaches position $\nu+1$ before going back to position $\nu-1$. Similarly, let $N_{\nu}^{-}$be the number of runs in which the cluster does the opposite, i.e., transfer from position $\nu$ to position $\nu-1$ before visiting position $\nu+1$. Also, let the number of those simulation runs in which, because of insufficiently long simulation time, the $n$-sized cluster becomes neither of size $n+x_{\nu}$ nor of size $n-x_{\nu-1}$ be ignored. Then $\lambda_{\nu}$ and $\mu_{\nu}$ are given by

$$
\begin{aligned}
& \lambda_{\nu}=N_{\nu}^{+} /\left(N_{\nu}^{+}+N_{\nu}^{-}\right), \\
& \mu_{\nu}=N_{\nu}^{-} /\left(N_{\nu}^{+}+N_{\nu}^{-}\right) .
\end{aligned}
$$

To express the growth probability $P(n)$ by means of $N_{\nu}^{+}$ and $N_{\nu}^{-}$we note that $P(n)$ is identical to the probability $P(\nu)$ for the $n$-sized cluster on position $\nu$ to reach position $w$ before going back to position 1. Accounting that the probabilities $\lambda_{\nu}$ and $\mu_{\nu}$ are analogous to the probabilities $p_{n}$ and $q_{n}$, this means that $P(n) \equiv P(\nu)$ is directly given by the right-hand side of Eq. (10) with $n$ and $M$ substituted by $\nu$ and $w$ and the ratio $g_{i} / f_{i}$ replaced by the ratio $N_{i}^{-} / N_{i}^{+}$. This is so, because in accordance with Eqs. (6), (7), (22), and (23) $g_{i} / f_{i}=q_{i} / p_{i}$ and $\mu_{i} / \lambda_{i}=N_{i}^{-} / N_{i}^{+}$. Thus $(2 \leqslant \nu \sigma \leqslant w-1)$

$$
\begin{aligned}
P(n)= & 1-\left[1+\sum_{i=2}^{w-1} N_{2}^{-} N_{3}^{-} \cdots N_{i}^{-} / N_{2}^{+} N_{3}^{+} \cdots N_{i}^{+}\right]^{-1} \\
& \times \sum_{i=\nu}^{w-1} N_{2}^{-} N_{3}^{-} \cdots N_{i}^{-} / N_{2}^{+} N_{3}^{+} \cdots N_{i}^{+}
\end{aligned}
$$

where $n$ is related to $\nu$ by

$$
n=1+\sum_{i=1}^{w-1} x_{i} .
$$

We note that the accuracy of the determination of $P(n)$ with the aid of Eq. (24) is better when the total number $N_{\nu}$ $=N_{\nu}^{+}+N_{\nu}^{-}$of simulation runs with the $n$-sized cluster is greater. Also, the error in the so-determined $P(n)$ depends on the lengths $x_{\nu}$ of the subintervals into which the interval $[1, M]$ is divided. At a fixed $N_{\nu}$, only at sufficiently large $x_{\nu}$ values the random walk of the $n$-sized cluster between sizes $n-x_{\nu-1}$ and $n+x_{\nu}$ is statistically significant enough to give $N_{\nu}^{-} / N_{\nu}^{+}$values with acceptable accuracy. When $x_{\nu}$ is chosen to be much less than the width $\Delta^{*}$ of the nucleus region, the $N_{\nu}^{-} / N_{\nu}^{+}$values obtained by the simulation may not be sufficiently accurate.

\section{SIMULATION DETAILS AND RESULTS}

Constant number of atoms, pressure and temperature Monte Carlo simulations ${ }^{19}$ were performed with an initially $n$-sized liquid cluster surrounded by a vapor subjected to periodic boundary conditions. The Lennard-Jones interaction potential was used with well depth $\varepsilon$ and atomic diameter $\sigma$. The potential was truncated and shifted at $r_{c}=2.5 \sigma$ and no long-range corrections were made. The simulations were done at temperature $T=0.741 \varepsilon / k$ and pressure $p$ $=0.016027 \varepsilon / \sigma^{3}$ resulting in supersaturation ratio $S=2.04$ (Ref. 6). On average, one volume change per $N$ atom displacements was performed where $N$ is the number of atoms in the simulation box $(900 \leqslant N \leqslant 970$ atoms $)$. The acceptance ratio of both the volume change and the atom displacement was kept at about $50 \%$.

In the simulations, in order to determine the cluster size a distinction has to be made between atoms belonging to the cluster and to the surrounding vapor. As done by ten Wolde and Frenkel, ${ }^{6}$ liquid atoms are defined to be atoms that have more than 4 neighboring atoms within a distance $r_{1}=1.5 \sigma$. Due to this definition of liquid atom, in the vapor occasionally liquid atoms appear which do not belong to the cluster and are, therefore, not counted when determining the cluster size. This is so, because a liquid atom belongs to the cluster only when within a distance $r_{1}=1.5 \sigma$ this atom has at least one liquid atom already belonging to the cluster.

A spherical liquid cluster was positioned in a sphere in the vapor from which the vapor atoms were removed making sure that there were no close contacts. A preliminary simulation run of at least 500000 steps was performed to bring the so-created system of cluster and vapor to the appropriate pressure and (local) density. This resulting configuration could then be used in the actual simulations. The final configurations in simulation runs with a growing or decaying $n$-sized cluster which ends up as a cluster of size $n+x_{\nu}$ or 
TABLE I. Simulation results from the first series.

\begin{tabular}{lllllll}
\hline \hline$\nu$ & $n$ & $N_{\nu}$ & $N_{\nu}^{+}$ & $N_{\nu}^{-}$ & $P(n)$ & $Q(n)$ \\
\hline 2 & 15 & 50 & 4 & 46 & 0.040 & 0.960 \\
3 & 45 & 33 & 17 & 16 & 0.497 & 0.503 \\
4 & 75 & 57 & 50 & 7 & 0.927 & 0.073 \\
5 & 105 & 18 & 15 & 3 & 0.987 & 0.013 \\
6 & 136 & 20 & 19 & 1 & 0.999 & 0.001 \\
7 & 165 & 20 & 20 & 0 & 1.000 & 0.000 \\
\hline \hline
\end{tabular}

$n-x_{\nu-1}$ could also be used as starting configurations. This was done in most of the simulations in which $x_{\nu}=30$ was chosen as the length of almost all size subintervals.

To determine the probabilities $\lambda_{\nu}$ and $\mu_{\nu}$ two to five different starting configurations of the $n$-sized cluster were used. For all these configurations at least 10 simulation runs were performed. During each run, after every 1000 simulation steps the cluster size was checked when it was sufficiently far away from the limiting values $n+x_{\nu}$ and $n$ $-x_{\nu-1}$. Upon approaching these values within 20 atoms the cluster size was determined after every 50 steps. Upon further approaching these values within 7 atoms the determination was done after each successful simulation step.

Two series of simulations with $M=195, w=8$, and $n$ $=15,45,75,105,136,165$ and with $M=210, w=8$, and $n=30,60,90,120,150,180$ were done. These $M$ values were chosen after it was established that clusters of size $n$ $>150$ practically never decayed to the monomer size $n=1$ and, hence, were large enough supernuclei. From the simulations the numbers $N_{\nu}^{+}$and $N_{\nu}^{-}$were determined. With these numbers, the values of $P(n)$ then follow from Eq. (24). Using this approach to determine $P(n)$ resulted in a significant reduction of the simulation time with respect to the time needed for the direct determination of $P(n)$ (in it the $n$-sized cluster is allowed to fully grow up to the maximum size $M$ or to completely decay to the monomer size 1$)$. The decay probability $Q(n)$ was obtained from Eq. (2) with the help of $P(n)$ already determined from Eq. (24).

The results from the two series of simulations are summarized in Tables I and II. The $P(n)$ dependence from these tables is depicted in Fig. 4 by the symbols (the circles and the triangles represent the $P(n)$ data from Table I and Table II, respectively). Application of the condition (21) to the data readily yields $n^{*} \approx 45$. In order to determine not only the nucleus size $n^{*}$, but also the quantity $\beta$, we can fit $P(n)$ from Eq. (18) to the simulation $P(n)$ data. The curve in Fig. 4 illustrates the best fit obtained with $n^{*}=47.8 \pm 1.0$ and $\beta$ $=0.0441 \pm 0.0040$. As seen, Eq. (18) describes well the

TABLE II. Simulation results from the second series.

\begin{tabular}{lllllll}
\hline \hline$\nu$ & $n$ & $N_{\nu}$ & $N_{\nu}^{+}$ & $N_{\nu}^{-}$ & $P(n)$ & $Q(n)$ \\
\hline 2 & 30 & 38 & 3 & 35 & 0.059 & 0.941 \\
3 & 60 & 35 & 26 & 9 & 0.748 & 0.252 \\
4 & 90 & 19 & 18 & 1 & 0.986 & 0.014 \\
5 & 120 & 20 & 18 & 2 & 0.999 & 0.001 \\
6 & 150 & 20 & 16 & 4 & 1.000 & 0.000 \\
7 & 180 & 19 & 18 & 1 & 1.000 & 0.000 \\
\hline \hline
\end{tabular}

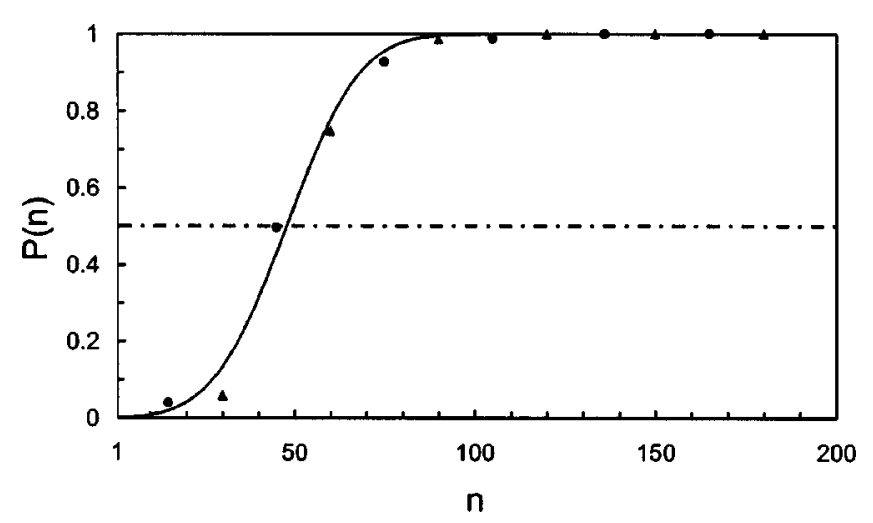

FIG. 4. Dependence of the cluster growth probability on the cluster size in homogeneous nucleation of droplets in Lennard-Jones vapors at $T$ $=0.741 \varepsilon / k$ and $S=2.04$ : Circles and triangles-Monte Carlo simulation data from Tables I and II, respectively; curve-best fit of Eq. (18).

simulation data. Using the found $\beta$ value, we can calculate the Zeldovich factor $z$ from Eq. (14): The result is $z$ $=0.0249$. As far as we know, this is the first determination of the Zeldovich factor by means of computer simulations. We note as well that the $n^{*}$ values resulting from the use of Eqs. (18) and (21) are practically the same. For comparison, ten Wolde and Frenkel ${ }^{6}$ found that $n^{*} \approx 60$ at $S=2.04$ when the nucleus is defined by the equimolecular dividing surface. The difference between their and our finding for $n^{*}$ at $S$ $=2.04$ may be attributed to the difference between the equimolecular dividing surface and the unknown dividing surface corresponding to the cluster definition used by us in the present simulation.

It is interesting to verify whether the $n^{*}$ and $\beta$ values obtained from the simulation are compatible with the classical relation (20). According to this relation, $6 \beta^{2} n^{*}=\ln S$. With $n^{*}=47.8$ and $\beta=0.0441$ from above it follows that $6 \beta^{2} n^{*}=0.558$ which compares with $\ln S=\ln 2.04=0.713$.

Finally, we can check whether the nucleus size determined in our simulation is predictable by the GibbsThomson Eq. (5). Using $\gamma=0.494 \varepsilon / \sigma^{2}$ for the specific surface energy of the planar Lennard-Jones liquid-gas interface, ${ }^{6} \rho=0.766 / \sigma^{3}$ for the molecular density of the bulk Lennard-Jones liquid at liquid-gas coexistence, ${ }^{6} T$ $=0.741 \varepsilon / k$ and $S=2.04$, we calculate $n^{*}=47$ which agrees with the simulation finding. This result is understandable in the light of a recent analysis ${ }^{20}$ revealing that, to a good approximation, Eq. (5) is applicable even to nuclei of a few atoms or molecules only.

\section{CONCLUSION}

The dependence of the cluster growth probability $P$ on the cluster size $n$ in the case of one-component nucleation of any kind is given exactly by the general Eqs. (10) or (11). The present analysis shows that to a very good approximation and without loss of generality $P(n)$ is expressed by Eq. (18). According to Eq. (18), the $P(n)$ dependence is controlled by two parameters only-the nucleus size $n^{*}$ and the Zeldovich factor $z$ [because $z$ is related to the quantity $\beta$ by Eq. (14)]. Available $P(n)$ data thus allow a model- 
independent determination of $n^{*}$ via Eq. (21) or of both $n^{*}$ and $z$ by treating $n^{*}$ and $\beta$ in Eq. (18) as fitting parameters.

The cluster growth probability $P(n)$ can be determined with the aid of computer simulations. This can be done either directly, i.e., by counting how many times the $n$-sized cluster reaches a previously specified large enough size before becoming a monomer, or indirectly, i.e., by means of Eq. (24) proposed in the paper. The use of this equation results in a great reduction of the computer time needed for the reliable determination of $P(n)$. Equation (24) is thus a valuable tool in computer simulations of one-component nucleation of any kind-homogeneous, heterogeneous, three-dimensional, or two-dimensional.

The analysis of the obtained simulation data for the growth probability of Lennard-Jones droplets in supersaturated Lennard-Jones vapors yields model-independent values for the nucleus size $n^{*}$ and the Zeldovich factor $z$. These values are in agreement with those predicted by the classical nucleation theory. The result for $n^{*}$ is another evidence for the applicability of the Gibbs-Thomson equation (5) to nuclei of nearly molecular size. A more comprehensive check of this applicability requires a determination of the supersaturation dependence of the nucleus size by simulations of the cluster growth probability at different supersaturations. Such simulations, as well as simulations with liquid or crystalline two- or three-dimensional clusters on solid substrates, are intended as the subject of a future work.

\section{ACKNOWLEDGMENT}

One of the authors (D. K.) gratefully acknowledges the hospitality that he enjoyed as a Visiting Professor at the Laboratory of Process Equipment of the Delft University of Technology.

${ }^{1}$ M. Volmer, Kinetik der Phasenbildung (Steinkopff, Dresden, 1939).

${ }^{2}$ S. Karlin, A First Course in Stochastic Processes (Academic, New York, 1966), p. 205.

${ }^{3}$ G. M. White, J. Chem. Phys. 50, 4672 (1969).

${ }^{4}$ D. I. Zhukhovitskii, J. Chem. Phys. 103, 9401 (1995).

${ }^{5}$ L. S. Bartell and J. Chen, J. Phys. Chem. 99, 12444 (1995).

${ }^{6}$ P. R. ten Wolde and D. Frenkel, J. Chem. Phys. 109, 9901 (1998).

${ }^{7}$ K. Yasuoka and M. Matsumoto, J. Phys. Chem. 109, 8451 (1998).

${ }^{8}$ I. Kusaka and D. W. Oxtoby, J. Phys. Chem. 110, 5249 (1999).

${ }^{9}$ K. J. Oh and X. C. Zeng, J. Phys. Chem. 110, 4471 (1999).

${ }^{10}$ K. J. Oh and X. C. Zeng, J. Chem. Phys. 112, 294 (2000).

${ }^{11}$ H. Vehkamaki and I. J. Ford, J. Chem. Phys. 112, 4193 (2000).

${ }^{12}$ S. Wonczak, R. Strey, and D. Stauffer, J. Chem. Phys. 113, 1976 (2000).

${ }^{13}$ K. Laasonen, S. Wonczak, R. Strey, and A. Laaksonen, J. Chem. Phys. 113, 9741 (2000).

${ }^{14}$ Y. G. Chushak and L. S. Bartell, J. Phys. Chem. A 104, 9328 (2000).

${ }^{15}$ Y. G. Chushak and L. S. Bartell, J. Phys. Chem. B 105, 11605 (2001).

${ }^{16}$ P. Schaaf, B. Senger, J.-C. Voegel, R. K. Bowles, and H. Reiss, J. Phys. Chem. 114, 8091 (2001).

${ }^{17}$ J. H. ter Horst, H. J. M. Kramer, and P. J. Jansens, Crystal Growth Design 2, 351 (2002).

${ }^{18}$ D. Kashchiev, Nucleation: Basic Theory with Applications (ButterworthHeinemann, Oxford, 2000).

${ }^{19}$ D. Frenkel and B. Smit, Understanding Molecular Simulation: from Algorithms to Applications (Academic, San Diego, 2002)

${ }^{20}$ D. Kashchiev, J. Chem. Phys. 118, 1837 (2003). 